\title{
LMIs-A Fundamental Tool in Analysis and Controller Design for Discrete Linear Repetitive Processes
}

\author{
K. Galkowski, Eric Rogers, S. Xu, J. Lam, Senior Member, and D. H. Owens
}

\begin{abstract}
Discrete linear repetitive processes are a distinct class of two-dimensional (2-D) linear systems with applications in areas ranging from long-wall coal cutting through to iterative learning control schemes. The feature which makes them distinct from other classes of 2-D linear systems is that information propagation in one of the two distinct directions only occurs over a finite duration. This, in turn, means that a distinct systems theory must be developed for them. In this paper, an LMI approach is used to produce highly significant new results on the stability analysis of these processes and the design of control schemes for them. These results are, in the main, for processes with singular dynamics and for those with so-called dynamic boundary conditions. Unlike other classes of 2-D linear systems, these feedback control laws have a firm physical basis, and the LMI setting is also shown to provide a (potentially) very powerful setting in which to characterize the robustness properties of these processes.
\end{abstract}

Index Terms-Linear matrix inequalities, repetitive processes, stability and control.

\section{INTRODUCTION}

$\mathbf{T}$ HE ESSENTIAL unique characteristic of a repetitive, or multipass process is a series of sweeps, termed passes, through a set of dynamics defined over a fixed finite duration known as the pass length. On each pass an output, termed the pass profile, is produced which acts as a forcing function on, and hence contributes to, the next pass profile. This, in turn, leads to the unique control problem for these processes in that the output sequence of pass profiles generated can contain oscillations that increase in amplitude in the pass to pass direction.

To introduce a formal definition, let $\alpha<+\infty$ denote the pass length (assumed constant). Then in a repetitive process the pass profile $y_{k}(t), 0 \leq t \leq \alpha$, generated on pass $k$ acts as a forcing

Manuscript received May 4, 2001; revised February 2, 2002. The work of K. Galkowski was supported by National Scientific Research Committee of Poland (KBN) under Grant 7T11A 020 20. The work of E. Rogers and D. H. Owens was supported by The Engineering and Physical Sciences Research Council. The work of S. Xu and J. Lam was supported by the Hong Kong University, Hong Kong under Grant CRCG 10202 466/19870. This paper was recommended by Co-Guest Editor M. N. S. Swamy.

K. Galkowski is with the Institute of Control and Computational Engineering, University of Zielona Gora, Zielona Gora 65-246, Poland.

E. Rogers is with the Department of Electronics and Computer Science, University of Southampton, Southampton SO17 1BJ, U.K.

$\mathrm{S}$. Xu is with the Department of Electrical and Computer Engineering, University of Alberta, Edmonton T6G 2V4, Canada.

J. Lam is with the Department of Mechanical Engineering, University of Hong Kong, Hong Kong.

D. H. Owens is with the Department of Automatic Control and Systems Engineering, University of Sheffield, Sheffield S1 3JD, U.K.

Publisher Item Identifier S 1057-7122(02)05601-5. function on the next pass and hence contributes to the dynamics of the new pass profile $y_{k+1}(t), 0 \leq t \leq \alpha, k \geq 0$.

Physical examples of repetitive processes include long-wall coal cutting and metal rolling operations (see, for example, [1]). Also in recent years applications have arisen where adopting a repetitive process setting for analysis has distinct advantages over alternatives. Examples of these so-called algorithmic applications of repetitive processes include classes of iterative learning control schemes [3], denoted by ILC in this paper, and iterative algorithms for solving nonlinear dynamic optimal control problems based on the maximum principle [4]. In the case of ILC for the linear dynamics case, the stability theory for so-called differential and discrete linear repetitive processes is the essential basis for a rigorous stability/convergence theory.

Attempts to control these processes using standard [or one-dimensional (1-D)] systems theory/algorithms fail (except in a few very restrictive special cases) precisely because such an approach ignores their inherent two-dimensional (2-D) systems structure, i.e., information propagation occurs from pass to pass and along a given pass. In seeking a rigorous foundation on which to develop a control theory for these processes it is natural to attempt to exploit structural links which exist between, in particular, the class of so-called discrete linear repetitive processes and 2-D linear systems described by the extensively studied Roesser [5] or Fornasini-Marchesini [6] state-space models. The discrete linear repetitive processes considered in this paper are distinct from such 2-D linear systems in the sense that information propagation in one of the two separate directions (along the pass) only occurs over a finite duration and hence large key elements of existing 2-D systems theory can either be: 1) not be applied, or 2) only applied after significant modifications.

The finite pass length is also the distinction between other classes of linear repetitive processes and the 2-D continuous-discrete linear systems studied by Kaczorek [7] and others. Also repetitive processes can exhibit very distinct dynamics depending on the structure of their initial, also termed boundary, conditions where, for example, incorrect representation of them can lead to incorrect stability conclusions (with obvious implications for subsequent analysis).

A rigorous stability theory for linear repetitive processes has been developed. This theory [8] is based on an abstract model in a Banach space setting which includes all such processes as special cases. Also the results of applying this theory to a wide range of cases have been reported, including the sub-class considered here. This has resulted in stability tests that can be im- 
plemented by direct application of well-known 1-D linear systems tests.

One critical area for these processes which has not received much attention to date is that of the specification and design of control schemes. The work already reported in this area has either focused on a particular application, e.g., ILC [3], where the control laws used are explicitly tailored to the needs of that area, or considered cases (see, for example, [2]) where strong structural constraints have been placed on the underlying process dynamics. Clearly, therefore, there is a need for a general theory which is applicable to the widest possible classes of dynamics and which results in systematic controller design procedures.

In this paper, we start from previous preliminary work to develop some highly significant new results in this key area using an LMI setting for analysis. Unlike other classes of 2-D linear systems (e.g., those described by the well known Roesser [5] or Fornasini-Marchesini [6] state-space models) the control laws considered have a well defined physical basis - a key issue in terms of eventual implementation. A major conclusion from the results in this paper is that the LMI setting is a powerful new tool for the analysis and, in particular, the specification and design of control schemes for discrete linear repetitive processes. This is based on the fact established for this first time in this paper that, in contrast to previous work/approaches, the LMI setting allows us to treat (either open loop or closed loop under allowable control law) the most general form of boundary conditions, uncertainty in the defining state-space model matrices, and singularity in the along the pass dynamics. The next section gives the required background results.

\section{BACKGROUND}

The state-space model of a discrete linear repetitive process has the following form over $0 \leq p \leq \alpha-1, k \geq 0$

$$
\begin{aligned}
x_{k+1}(p+1) & =A x_{k+1}(p)+B u_{k+1}(p)+B_{0} y_{k}(p) \\
y_{k+1}(p) & =C x_{k+1}(p)+D u_{k+1}(p)+D_{0} y_{k}(p) .
\end{aligned}
$$

Here, on pass $k, x_{k}(p)$ is the $n \times 1$ state vector, $y_{k}(p)$ is the $m \times 1$ vector pass profile, and $u_{k}(p)$ is the $r \times 1$ vector of control inputs. To complete the process description, it is necessary to specify the boundary conditions, i.e., the initial pass profile vector $y_{0}(p)$ and the pass state initial vector sequence $\left\{x_{k}(0)\right\}_{k \geq 1}$. The simplest possible form of these is where, in particular, the pass state initial vector sequence is independent of the previous pass profile dynamics. Such conditions take the form

$$
\begin{aligned}
x_{k+1}(0) & =d_{k+1}, & & k \geq 0 \\
y_{0}(p) & =f(p), & & 0 \leq p \leq \alpha-1
\end{aligned}
$$

where $d_{k+1}$ is an $n \times 1$ vector with known constant entries and the entries in the $m \times 1$ vector $f(p)$ are known functions of $p$.

In some cases, the boundary conditions (2) are simply not strong enough to 'adequately' model the underlying dynamics - even for preliminary simulation/control analysis. For example, the optimal control application [4] requires the use of pass state initial vectors which are a function of the previous pass profile. Hence the structure of (2) must be extended to include this case (and others).

In terms of analysis, it is clearly of prime importance to start with the a general form of boundary conditions with subsequent specialization to particular cases as required. Other work [2] has concluded that the most general set results from replacing $x_{k+1}(0), k \geq 0$, in (2) by

$$
x_{k+1}(0)=d_{k+1}+\sum_{p=0}^{\alpha-1} J_{p} y_{k}(p)
$$

where $J_{p}, 0 \leq p \leq \alpha-1$, are $n \times m$ matrices with constant entries. For ease of terminology, we will refer to the constant pass state vector sequence of (2) as 'static' and those of the form of (3) as 'dynamic' (to signify that in the former case they are independent of the previous pass profile and (explicitly) dependent in the latter case).

In the state-space model of (1), the current pass (state and pass profile) dynamics only (explicitly) depend on the previous pass profile and, as such, are termed unit memory. More generally, a discrete nonunit memory linear repetitive process with memory length $M>1$ is described by the following state-space model over $0 \leq p \leq \alpha-1, k \geq 0$

$$
\begin{aligned}
x_{k+1}(p+1) & =A x_{k+1}(p)+B u_{k+1}(p)+\sum_{j=0}^{M-1} B_{j} y_{k-j}(p) \\
y_{k+1}(p) & =C x_{k+1}(p)+D u_{k+1}(p)+\sum_{j=0}^{M-1} D_{j} y_{k-j}(p) .
\end{aligned}
$$

This model clearly reduces to (1) when $M=1$ (termed the unit memory case) and can be subject to boundary conditions which are the natural generalization of (2) or (3) (and hence are not explicitly detailed at this stage).

The abstract model-based stability theory for linear constant pass length repetitive processes consists of the distinct concepts of so-called asymptotic and stability along the pass respectively. Recognizing the unique control problem for these processes, asymptotic stability demands a form of bounded input bounded output (BIBO) stability over the (finite and constant) pass length $\alpha$. In particular, this property is defined in terms of the norm on the underlying function space and it holds provided bounded sequences of inputs (on each pass, the corresponding element in this sequence is formed the state initial conditions on this pass and any control inputs and disturbances which are applied on it) produce bounded sequences of pass profiles. If this is indeed the case, then the resulting pass profile sequence is guaranteed to converge to a steady or so-called limit profile which for all cases considered here is described by a 1-D discrete linear systems state-space model.

The fact that the pass length is finite and constant means that this limit profile may not have acceptable along the pass dynamics, where the most basic requirement is stability of the limit profile as a 1-D discrete linear system, i.e., all eigenvalues of the state matrix have modulus strictly less than unity. (Examples are easily generated to highlight this fact). Hence, in general, it will be the stronger property of stability along the pass which will be 
required as it prevents this problem from arising by demanding the BIBO property uniformly, i.e., with respect to the pass length $\alpha$.

In the case of processes described by (1) and (2), several equivalent sets of necessary and sufficient conditions for stability along the pass have been reported (see, for example, [8]) but here it is the following set which is required.

Theorem 1: Discrete linear repetitive processes described by (1) and (2) are stable along the pass if, and only if, the 2D characteristic polynomial

$$
\begin{aligned}
C\left(z_{1}, z_{2}\right):=\operatorname{det}\left[\begin{array}{cc}
I_{n}-z_{1} A & -z_{1} B_{0} \\
-z_{2} C & I_{m}-z_{2} D_{0}
\end{array}\right] & \neq 0 \\
\forall\left(z_{1}, z_{2}\right) & \in \bar{U}^{2}
\end{aligned}
$$

where $\bar{U}^{2}=\left\{\left(z_{1}, z_{2}\right):\left|z_{1}\right| \leq 1,\left|z_{2}\right| \leq 1\right\}$.

Suppose now that $r(\cdot)$ denotes the spectral radius of its argument, i.e., in the case (as in this paper) of a matrix compute its set of eigenvalues and then compute the largest modulus of the members of this set. Then, (5) gives the necessary conditions that $r\left(D_{0}\right)<1$ (asymptotic stability) and $r(A)<1$ (essentially the dynamics along the first pass are uniformly bounded with respect to the pass length) which should be verified before proceeding further with any stability analysis. (Also, asymptotic stability for all possible values of the pass length is a necessary condition for stability along the pass.) The conditions for asymptotic stability and stability along the pass of (1) with boundary conditions (3) for all cases treated in this paper will be given in context later as it develops.

The following result is crucial to the analysis in this paper. This result is very well known and its proof can, for example, be found in [9].

Lemma 1: Given constant matrices $W, L, V$ of appropriate dimensions where $W=W^{T}$ and $V=V^{T}>0$, then $W+$ $L^{T} V L<0$ if, and only if

$$
\left[\begin{array}{cc}
W & L^{T} \\
L & -V^{-1}
\end{array}\right]<0
$$

The matrix $W+L^{T} V L$ is known as the Schur complement of $V$.

\section{BASIC LMI STABILITY ANALYSIS}

To express the result of Theorem 1 in LMI terms, define the following matrices from the state-space model of (1)

$$
\hat{A}_{1}=\left[\begin{array}{cc}
A & B_{0} \\
0 & 0
\end{array}\right], \quad \hat{A}_{2}=\left[\begin{array}{cc}
0 & 0 \\
C & D_{0}
\end{array}\right] .
$$

Then, we have the following sufficient condition for stability along the pass of processes described by (1) and (2).

Theorem 2: Discrete linear repetitive processes described by (1) and (2) are stable along the pass if $\exists$ symmetric matrices $P>0$ and $Q>0$ satisfying the following LMI

$$
\left[\begin{array}{cc}
\hat{A}_{1}^{T} P \hat{A}_{1}+Q-P & \hat{A}_{1}^{T} P \hat{A}_{2} \\
\hat{A}_{2}^{T} P \hat{A}_{1} & \hat{A}_{2}^{T} P \hat{A}_{2}-Q
\end{array}\right]<0 .
$$

Proof: This is, in effect, a proof that the LMI condition of (7) is a sufficient condition for (5) to hold and proceeds as follows.

The first step is to note that by use of Lemma 1, (7) is equivalent to

$Q-\hat{A}_{2}^{T} P \hat{A}_{2}>0$

and

$\hat{A}_{1}^{T} P \hat{A}_{1}+\hat{A}_{1}^{T} P \hat{A}_{2}\left(Q-\hat{A}_{2}^{T} P \hat{A}_{2}\right)^{-1} \hat{A}_{2}^{T} P \hat{A}_{1}+Q-P<0$.

Also, it is easy to show that $\forall\left|z_{2}\right| \leq 1$

$$
Q-\left|z_{2}\right|^{2} \hat{A}_{2}^{T} P \hat{A}_{2}>0
$$

and hence (using this last fact and (9)) we have that $\forall\left(z_{1}, z_{2}\right) \in$ $\bar{U}^{2}$

$$
\begin{aligned}
\left|z_{1}\right|^{2} \hat{A}_{1}^{T} P \hat{A}_{1}+\left|z_{1}\right|^{2}\left|z_{2}\right|^{2} \hat{A}_{1}^{T} P \hat{A}_{2}\left(Q-\left|z_{2}\right|^{2} \hat{A}_{2}^{T} P \hat{A}_{2}\right)^{-1} \\
\times \hat{A}_{2}^{T} P \hat{A}_{1}+Q-P<0
\end{aligned}
$$

It is a standard fact that for any two matrices $M$ and $N$ of the same dimensions, $M+M^{*} \leq N+M^{*} N^{-1} M$, where $*$ denotes the complex conjugate operation. Setting $M=z_{1}^{*} z_{2} \hat{A}_{2}^{T} P \hat{A}_{1}$, $N=Q-\left|z_{2}\right|^{2} \hat{A}_{2}^{T} P \hat{A}_{2}$, we conclude from (11) that $\forall\left(z_{1}, z_{2}\right) \in$ $\bar{U}^{2}$

$$
\left(z_{1} \hat{A}_{1}+z_{2} \hat{A}_{2}\right)^{*} P\left(z_{1} \hat{A}_{1}+z_{2} \hat{A}_{2}\right)-P<0
$$

i.e., $r\left(z_{1} \hat{A}_{1}+z_{2} \hat{A}_{2}\right)<1, \forall\left(z_{1}, z_{2}\right) \in \bar{U}^{2}$. This, in turn, implies that

$$
\operatorname{det}\left(I_{n+m}-z_{1} \hat{A}_{1}-z_{2} \hat{A}_{2}\right) \neq 0 \quad \forall\left(z_{1}, z_{2}\right) \in \bar{U}^{2}
$$

which is equivalent to (5) and hence the proof is complete.

In recent work, [2], the following result has been established.

Theorem 3: Discrete linear repetitive processes described by (1) and (2) are stable along the pass if $\exists$ symmetric matrices $W_{1}>0, W_{2}>0$ and $\hat{Q}>0$ such that the so-called 2-D Lyapunov equation

$$
W-\hat{A}^{T} W \hat{A}=\hat{Q}
$$

holds, where $\hat{A}:=\hat{A}_{1}+\hat{A}_{2}$ is the so-called augmented plant matrix and $W=\operatorname{diag}\left\{W_{1}, W_{2}\right\}$.

Hence, it follows immediately that the 2D Lyapunov condition for stability along the pass also has an LMI interpretation. This, in turn, means that the performance information [2] available for discrete linear repetitive processes via this last theorem is also available in the LMI setting.

\section{EXTENSIONS OF THE BASIC LMI-BASED STABILITY ANALYSIS}

The stability result (Theorem 2) is perhaps not that surprising given very similar results already available in the $2 \mathrm{D}$ linear systems area in general-see, for example, [10]. For discrete linear repetitive processes, however, there is a need to deal with the dynamic boundary conditions and other forms of dynamics such as those which are singular along the pass 
(in a form which will be made precise later). In this section we develop new results which show that the LMI approach can be applied to all relevant cases of the detailed dynamic structure of (unit and nonunit memory) discrete linear repetitive processes with dynamic boundary conditions, and also singular along the pass dynamics. This is in contrast to previously reported results where different approaches had to be applied to the various cases in order to get tractable stability tests. Also, it will be subsequently shown that the LMI approach, again in contrast to other approaches, provides a 'sufficiently tractable' basis on which to answer (in the main) currently open questions on the structure and design of control schemes. In particular, we will show how a powerful form of feedback control law (a combination of current pass state feedback and feedforward action based on the previous pass profile) can be designed using the LMI approach without the need to impose restrictions on the open loop process dynamics (including the form of the boundary conditions.) Moreover, we will also show that the LMI setting is a (potentially) very powerful method for addressing stability analysis and controller design for cases where there is uncertainty associated with the process dynamics - a critical area for which no substantial results are currently available.

\section{A. Dynamic Boundary Conditions}

Consider the case of unit memory processes with the dynamic boundary conditions of the form of (3). Then clearly an extension of the results given in the previous section would prove 'very difficult' (if not impossible). As an alternative, we proceed via the equivalent 1-D discrete linear systems state-space model of the underlying dynamics developed in [11]. A key feature of this 1-D equivalent model is that it is (unlike other 1-D equivalent models for 2-D linear systems, such as those for Roesser/Foransini-Marchesini state-space model structures [12]) defined in terms of vectors whose dimensions remain constant as the process evolves and block matrix elements with constant entries defined in terms of the matrices in (1) and (3).

To give the basic form of the 1-D equivalent model, first introduce $v_{k}(p):=y_{k-1}(p), 0 \leq p \leq \alpha-1, l:=k+1$, and define the so-called global pass profile, state and input super-vectors, respectively, for (1) as

$$
\begin{aligned}
& \mathbf{Y}(l):=\left[\begin{array}{c}
v_{l}(0) \\
v_{l}(1) \\
\vdots \\
v_{l}(\alpha-1)
\end{array}\right] \\
& \mathbf{X}(l):=\left[\begin{array}{c}
x_{l}(1) \\
x_{l}(2) \\
\vdots \\
x_{l}(\alpha)
\end{array}\right] \\
& \mathbf{U}(l):=\left[\begin{array}{c}
u_{l}(0) \\
u_{l}(1) \\
\vdots \\
u_{l}(\alpha-1)
\end{array}\right]
\end{aligned}
$$

Then, the 1-D equivalent model is given by

$$
\begin{aligned}
\mathbf{Y}(l+1) & =\Phi \mathbf{Y}(l)+\Delta \mathbf{U}(l)+\Theta d_{l} \\
\mathbf{X}(l) & =\Gamma \mathbf{Y}(l)+\Sigma \mathbf{U}(l)+\Psi d_{l}
\end{aligned}
$$

and the defining matrices are constructed from appropriate entries of the matrices which define (1) and (3). For the purposes of stability analysis, however, it is only the detailed structures of $\Phi$ and $\Delta$ which are required, as shown in (16) and (17) at the bottom of the page.

The following result [11], [13] gives a necessary and sufficient condition for asymptotic stability in this case.

Theorem 4: Discrete linear repetitive processes described by (1) and (3) are asymptotically stable if, and only if, $r(\Phi)<1$.

Also, the extra condition for stability along the pass is that the 2D characteristic polynomial $C\left(z_{1}, z_{2}\right)$ satisfies (5) and hence this condition can also be dealt with by the LMI based analysis of Section III. Note also that a necessary and sufficient condition for Theorem 4 to hold is (using the 1-D discrete linear systems Lyapunov equation) that $\exists$ a symmetric matrix $W>0$ such that the following LMI is satisfied (we will make use of this result later in the paper)

$$
\left[\begin{array}{cc}
-W & \Phi^{T} W \\
W \Phi & -W
\end{array}\right]<0
$$

$$
\begin{aligned}
& \Phi=\left[\begin{array}{cccc}
D_{0}+C J_{0} & C J_{1} & \cdots & C J_{\alpha-1} \\
C\left(B_{0}+A J_{0}\right) & \left(D_{0}+C A J_{1}\right) & \cdots & C A J_{\alpha-1} \\
C A\left(B_{0}+A J_{0}\right) & C\left(B_{0}+A^{2} J_{1}\right) & \cdots & C A^{2} J_{\alpha-1} \\
\vdots & \vdots & \ddots & \vdots \\
C A^{\alpha-2}\left(B_{0}+A J_{0}\right) & C A^{\alpha-3}\left(B_{0}+A^{2} J_{1}\right) & \cdots & \left(D_{0}+C A^{\alpha-1} J_{\alpha-1}\right)
\end{array}\right] \\
& \Delta=\left[\begin{array}{ccccc}
D & 0 & 0 & \cdots & 0 \\
C B & D & 0 & \cdots & 0 \\
C A B & C B & D & \cdots & 0 \\
\vdots & \vdots & \vdots & \ddots & \vdots \\
C A^{\alpha-2} B & C A^{\alpha-3} B & C A^{\alpha-4} & \cdots & D
\end{array}\right]
\end{aligned}
$$


Consider now the nonunit memory case. Then, the natural generalization of the boundary conditions of (2) for this case is

$$
\left.\begin{array}{l}
x_{k+1}(0)=d_{k+1}, \quad k \geq 0 \\
y_{-j}(p)=f_{-j}(p), \quad 0 \leq p \leq \alpha-1, \quad 0 \leq j \leq M-1
\end{array}\right\} .
$$

Now, introduce the substitutions $l=k+1$ and $y_{l}(p):=$ $v_{l+1}(p), 0 \leq p \leq \alpha-1, l=2-M, 1-M, \ldots, 0,1, \ldots$. Then, the dynamics of discrete nonunit memory linear repetitive processes described by (4) and (19) are equivalently described by

$$
\begin{gathered}
\left.x_{l}(p+1)=A x_{l}(p)+B u_{l}(p)+\sum_{j=0}^{M-1} B_{j} v_{l-j}(p)\right\} \\
v_{l+1}(p)=C x_{l}(p)+D u_{l}(p)+\sum_{j=0}^{M-1} D_{j} v_{l-j}(p)
\end{gathered}
$$

where $l \geq 1,0 \leq p \leq \alpha-1$, and boundary conditions are

$$
\begin{aligned}
& x_{l}(0)=d_{l}, \quad l \geq 1 \\
& v_{j}(p)=f_{j}(p), \quad 0 \leq p \leq \alpha-1, \quad 2-M \leq j \leq 1 .
\end{aligned}
$$

In the case of the dynamic boundary conditions for nonunit memory processes, the natural generalization of (3) is

$$
x_{k+1}(0)=d_{k+1}+\sum_{j=0}^{M-1} \sum_{p=0}^{\alpha-1} J_{j p} y_{k-j}(p), \quad k \geq 0 .
$$

It now follows immediately that the 1-D equivalent model-based analysis given above generalizes in a natural manner to this case and hence the details are omitted here. Later in the paper we will develop the LMI based asymptotic stability condition (18) for this case into an equivalent result which is numerically more tractable for both open loop and closed loop (under a suitably defined control law). Next, we give the first substantial results on the stability of processes with singular dynamics along the pass. Here again we will see the power and versatility of the LMI based approach.

\section{B. Processes With Singular Dynamics}

In what follows, we extend the results given so far in this paper to the case of discrete linear repetitive processes of the form (1) when the dynamics along the pass are singular (in the form made precise below). For brevity, we only consider unit memory processes of the form described by (1) and (2), where the latter are taken as zero without loss of generality. This is because the analysis which follows can be generalized in a natural manner to all other processes considered in this paper.

As starting point, we require the following well known result for singular 1-D discrete linear systems with state-space model

$$
E x(p+1)=A x(p)+B u(p)
$$

where $x$ denotes the $n \times 1$ state vector and $u$ denotes the $r \times 1$ vector of control inputs. The matrix $E$ is singular, i.e., $\operatorname{rank} E=h<n$. Also, see e.g., [14], such systems are termed impulse-free if the degree of $\operatorname{det}(s E-A)$ is equal to $\operatorname{rank} E$, and regular if $\operatorname{det}(s E-A)$ is not identically zero.
Lemma 2: The following hold in respect of systems described by (23).

1) For any regular, impulse-free pair $(E, A) \exists$ two real nonsingular matrices $S$ and $T$ such that

$$
S E T=\left[\begin{array}{cc}
I_{h} & 0 \\
0 & 0
\end{array}\right] \quad S A T=\left[\begin{array}{cc}
A_{1} & 0 \\
0 & I_{n-h}
\end{array}\right] .
$$

2) A descriptor system of the form (23) is stable if and only if the sub-matrix $A_{1}$ in the above decomposition is stable.

The singular discrete linear repetitive processes considered in this paper have a somewhat similar structure to (23) in the sense that the current pass state dynamics are governed by

$$
E x_{k+1}(p+1)=A x_{k+1}(p)+B u_{k+1}(p)+B_{0} y_{k}(p)
$$

with singular matrix $E$. Hence the dynamics here are singular along the pass. Note also we could consider cases where the dynamics are singular from pass to pass but such processes are of little interest in terms of applications.

Suppose now that the matrix $E$ has rank $h$, where $h<n$. Then, the following result extends Lemma 2 to discrete linear repetitive processes with dynamics which are singular along the pass in the sense defined by (24).

Lemma 3: Suppose that the matrix $E$ has rank $h$, and that the pair $(E, A)$ is regular and impulse-free. Then, $\exists$ square nonsingular matrices $T$ and $S$ such that

$$
\begin{aligned}
S E T & =\left[\begin{array}{cc}
I_{h} & 0 \\
0 & 0
\end{array}\right] \\
S A T & =\left[\begin{array}{cc}
A_{1} & 0 \\
0 & I_{n-h}
\end{array}\right] \\
S B_{0} & =\left[\begin{array}{l}
B_{01} \\
B_{02}
\end{array}\right] \\
S B & =\left[\begin{array}{l}
B_{1} \\
B_{2}
\end{array}\right]
\end{aligned}
$$

Proof: This is a straightforward consequence of Lemma 2 and hence the details are omitted here.

Applying these transforms to the discrete linear repetitive process state equation of (24) is equivalent to introducing the new state variable

$$
\tilde{x}_{k}(p)=T^{-1} x_{k}(p):=\left[\begin{array}{l}
\omega_{k}(p) \\
\sigma_{k}(p)
\end{array}\right]
$$

where $\omega_{k}(p) \in \mathbb{R}^{h}, \sigma_{k}(p) \in \mathbb{R}^{n-h}$ and left multiplying (24) by $S$. This gives the complete state-space model (i.e., the result of transforming (24) and the pass profile update equation) of a singular along the pass discrete linear repetitive process as

$$
\begin{aligned}
\omega_{k+1}(p+1)= & A_{1} \omega_{k+1}(p)+B_{1} u_{k+1}(p)+B_{01} y_{k}(p) \\
0= & \sigma_{k+1}(p)+B_{2} u_{k+1}(p)+B_{02} y_{k}(p) \\
y_{k+1}(p)= & C_{1} \omega_{k+1}(p)+C_{2} \sigma_{k+1}(p) \\
& +D u_{k+1}(p)+D_{0} y_{k}(p)
\end{aligned}
$$

where $\left[C_{1} C_{2}\right]=C T$.

Hence, on solving (26) for $\sigma_{k+1}(p)$ and inserting the result in (27) yields the equivalent model of the form (1) as (25) de- 
scribing the current pass state dynamics, and the current pass profile dynamics described by

$$
y_{k+1}(p)=C_{1} \omega_{k+1}(p)+\tilde{D} u_{k+1}(p)+\tilde{D}_{0} y_{k}(p)
$$

where $\tilde{D}=D-C_{2} B_{2}, \tilde{D}_{0}=D_{0}-C_{2} B_{02}$. Hence, we see that the static part of the along the pass dynamics of a discrete linear repetitive process with singular dynamics has a very significant effect on the overall process dynamics. This is evident in the following result which is simply Theorem 1 applied to the discrete linear repetitive process with current pass state dynamics described by (25) and current pass profile dynamics by (28).

Theorem 5: Discrete linear repetitive processes described by (25) and (28) with boundary conditions (2) are stable along the pass if, and only if

$$
\begin{aligned}
C^{\prime}\left(z_{1}, z_{2}\right):=\operatorname{det}\left[\begin{array}{cc}
I_{n}-z_{1} A_{1} & -z_{1} B_{01} \\
-z_{2} C_{1} & I_{m}-z_{2} \tilde{D}_{0}
\end{array}\right] \neq 0 \\
\forall\left(z_{1}, z_{2}\right) \in \bar{U}^{2} .
\end{aligned}
$$

In order to apply the results of Theorem 5 to a given example, it is first necessary to compute the decomposition (25)-(27) and hence (28). Then, the result of Theorem 2 can be used to obtain an LMI based sufficient condition for stability along the pass. Note also that the only requirement here is that the required decomposition actually exists.

For numerical work, note that the essential step of obtaining (25) and (28) could be numerically ill-conditioned. Hence it would be of considerable benefit if it were possible to obtain a set of necessary and sufficient conditions for stability along the pass which avoided this step. Here this is left as an area for further research with the note that the natural combination of Theorems 2 and 4 when the matrices $P$ and $Q$ do not have any structure imposed on them (apart from being symmetric and positive definite) is not possible.

It is possible to generalize the approach of [2] and, in particular Theorem 3 here, which employs a block diagonal matrix $W$, to the singular case. The actual result is as follows.

Theorem 6: Consider singular discrete linear repetitive processes described by (25) and (28) with boundary conditions (2). Then such processes are stable along the pass if the following LMI is feasible

$$
\begin{aligned}
& \hat{A}^{T} W \hat{A}<\tilde{E}^{T} W \tilde{E} \\
& \tilde{E}^{T} W \tilde{E} \geq 0
\end{aligned}
$$

where the symmetric matrix $W=\operatorname{diag}\left\{W_{1}, W_{2}\right\}$, and $\tilde{E}=$ $\operatorname{diag}\{E, I\}$.

Proof: Consider (29) and (30) applied to the decomposed model of (25)-(28), i.e., (with a compatible decomposition of $W_{1}$ and writing $W_{33}=W_{2}$ )

$$
\begin{gathered}
{\left[\begin{array}{ccc}
A_{1}^{T} & 0 & C_{1}^{T} \\
0 & I_{n-h} & C_{2}^{T} \\
B_{01}^{T} & B_{02}^{T} & D_{0}^{T}
\end{array}\right]\left[\begin{array}{ccc}
W_{11} & W_{12} & 0 \\
W_{12}^{T} & W_{22} & 0 \\
0 & 0 & W_{33}
\end{array}\right]} \\
\times\left[\begin{array}{ccc}
A_{1} & 0 & B_{01} \\
0 & I_{n-h} & B_{02} \\
C_{1} & C_{2} & D_{0}
\end{array}\right]<\left[\begin{array}{ccc}
W_{11} & 0 & 0 \\
0 & 0 & 0 \\
0 & 0 & W_{33}
\end{array}\right]
\end{gathered}
$$

$$
\hat{W}_{1}=\left[\begin{array}{cc}
W_{11} & 0 \\
0 & W_{33}
\end{array}\right] \geq 0
$$

Also introduce

$$
V=\left[\begin{array}{ccc}
I_{h} & 0 & 0 \\
0 & -B_{02} & I_{n-h} \\
0 & I_{m} & 0
\end{array}\right]
$$

and then pre- and post-multiply (30) by $V$ and its transpose respectively to obtain the equivalent condition

$$
\begin{aligned}
{\left[\begin{array}{cc}
\tilde{A}_{1}^{T} \hat{W}_{1} \tilde{A}_{1} & \star \\
\star & W_{22}+C_{2}^{T} W_{33} C_{2}
\end{array}\right] } & <\left[\begin{array}{cc}
W_{1} & 0 \\
0 & 0
\end{array}\right] \\
\tilde{A} & =\left[\begin{array}{cc}
A_{1} & B_{01} \\
C & \tilde{D}_{0}
\end{array}\right]
\end{aligned}
$$

and $\star$ denotes a submatrix block of compatible dimensions whose exact structure is not relevant to what follows.

Now, we can select $\varepsilon>0$ such that the following two conditions simultaneously hold

$$
\left.\begin{array}{cc}
{\left[\begin{array}{cc}
W_{11} & 0 \\
0 & W_{33}
\end{array}\right]+\varepsilon I>0} \\
\tilde{A}^{T}\left(\hat{W}_{1}+\varepsilon I\right) \tilde{A} & \star \\
\star & W_{22}+C_{2}^{T} W_{33} C_{2}
\end{array}\right]<\left[\begin{array}{cc}
\hat{W}_{1}+\varepsilon I & 0 \\
0 & 0
\end{array}\right] .
$$

This is the equivalent LMI condition to (13) of Theorem 3 for this case and the proof is complete.

\section{LMI BASED CONTROLLER DESIGN-STATIC BOUNDARY CONDITIONS}

In terms of the design of control schemes, it is instructive to consider first the case of static boundary conditions for unit memory processes since the results obtained generalize in a natural manner to the case of dynamic boundary conditions. Also, as shown later, further analysis is possible in this latter case.

In terms of the design of control schemes for discrete linear repetitive processes, most of the reported work has been done in the ILC area where it has become clear that the most powerful control action comes from feedback action on the current pass augmented by feedforward action from the previous pass (or the previous $M$ passes in the non unit memory case). Here, we consider a control law of the following form over $0 \leq p \leq$ $\alpha-1, k \geq 0$

$$
u_{k+1}(p)=K_{1} x_{k+1}(p)+K_{2} y_{k}(p):=K\left[\begin{array}{c}
x_{k+1}(p) \\
y_{k}(p)
\end{array}\right]
$$

where $K_{1}$ and $K_{2}$ are appropriately dimensioned matrices to be designed. Suppose also that this control law is applied to processes described by (1) and (2). Then, applying Theorem 1 to the resulting state-space model gives the necessary and sufficient condition for stability along the pass as shown in (37) at the bottom of the next page.

At this stage, we can give the following result which provides easily verified necessary conditions for the existence of a control law of the form (36) which guarantees closed loop stability along the pass. The proof of this result is obvious from (37) and is hence omitted here. 
Lemma 4: The following three conditions are necessary for stability along the pass of processes described by (1) and (2) under a control law of the form (36).

a) $(A, B)$ as a 1-D discrete linear system (i.e., state matrix $A$ and input matrix $B$ ) can be stabilized closed loop by a state feedback control law.

b) $\left(D_{0}, D\right)$ as a 1-D linear system (i.e., state matrix $D_{0}$ and input matrix $D$ ) can be stabilized closed loop by a state feedback control law.

c) $(\hat{A}, \hat{B})$ as a $1-\mathrm{D}$ linear system (i.e., state matrix $\hat{A}$ and input matrix $\hat{B}$ ) can be stabilized closed loop by a state

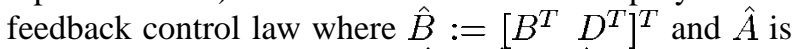
the augmented plant matrix $\left(\hat{A}=\hat{A}_{1}+\hat{A}_{2}\right)$.

Clearly, these conditions should be verified before proceeding further with a given design example.

It can be shown [8] that processes described by (1) and (2) are stable along the pass if, and only if, $r\left(D_{0}\right)<1, r(A)<1$, and all eigenvalues of the transfer function matrix

$$
G\left(z_{1}\right)=C\left(z_{1} I_{n}-A\right)^{-1} B_{0}+D_{0}
$$

have modulus strictly less than unity $\forall\left|z_{1}\right|=1$. Hence we have the following set of necessary and sufficient conditions for closed loop stability along the pass.

Lemma 5: Discrete linear repetitive processes described by (1) and (2) are stable along the pass under the action of the control law (36) if, and only if :

a) $A+B K_{1}$ and $D_{0}+D K_{2}$ are 1-D linear systems stable; and

b) $\left(C+D K_{1}\right)\left(z_{1} I_{n}-\left(A+B K_{1}\right)\right)^{-1}\left(B_{0}+B K_{2}\right)+D_{0}+$ $D K_{2}$ with $\left|z_{1}^{-1}\right| \leq 1$ is 1 -D linear systems stable.

This shows that designing (36) to ensure stability along pass when the resulting control law is applied to processes described by (1) and (2) can be reduced to applying the 1-D pole placement problem (twice) for 1-D discrete linear time invariant systems and then stabilizing another 1-D system with a complex parameter.

This latter problem has been the subject of work over the years in a number of areas and one approach is based on first re-writing condition (b) above as the requirement that $F\left(z_{1}^{-1}\right)+$ $M_{1}\left(z_{1}^{-1}\right) K_{2}$ with $\left|z_{1}^{-1}\right| \leq 1$ is 1-D stable, where

$$
\begin{aligned}
& F\left(z_{1}^{-1}\right)=D_{0}+P\left(z_{1}^{-1}\right) B_{0} \\
& M_{1}\left(z_{1}^{-1}\right)=D+P\left(z_{1}^{-1}\right) B
\end{aligned}
$$

and

$$
P\left(z_{1}\right)=z_{1}^{-1} \frac{\left(C+D K_{1}\right) \operatorname{adj}\left(I_{n}-z_{1}^{-1}\left(A+B K_{1}\right)\right)}{\operatorname{det}\left(I_{n}-z_{1}^{-1}\left(A+B K_{1}\right)\right)}
$$

Now write

$$
R=\left\{\frac{b\left(z_{1}^{-1}\right)}{a\left(z_{1}^{-1}\right)}: a\left(z_{1}^{-1}\right) \neq 0,\left|z_{1}^{-1}\right| \leq 1\right\}
$$

where $a\left(z_{1}^{-1}\right)$ and $b\left(z_{1}^{-1}\right)$ are polynomials in $z_{1}^{-1}$ with real coefficients. This then means that $R$ is a principal integral domain and suppose that $F$ and $G$ are matrices with real entries and of dimensions $d \times d$ and $d \times h$, respectively. Then, it is a standard fact that if the pair $(F, G)$ is $R$-reachable, i.e., every $x \in R^{d}$ is an $R$-linear combination of the columns of $G, F G, \ldots, F^{d-1} G$, then for every $\lambda_{i}, 1 \leq i \leq d \in R, \exists \hat{K}$ of dimension $h \times d$ such that

$$
\operatorname{det}\left(z I_{d}-(F+G \hat{K})\right)=\prod_{i=1}^{d}\left(z-\lambda_{i}\right)
$$

These facts and Lemma 5 lead to the following.

Theorem 7: Discrete linear repetitive processes described by (1) and (2) are stable along the pass under the action of the control law (36) with gain matrix $K=\left[K_{1}^{T} K_{2}^{T}\left(z_{1}^{-1}\right)\right]^{T}$ where $K_{1}$ is an $r \times n$ matrix and $K_{2}\left(z_{1}^{-1}\right) \in R^{r \times m}$ if:

a) $(A, B)$ as a 1-D discrete linear system is stabilized closed loop by a state feedback control law with gain matrix $K_{1}$.

b) For some $K_{1}$ stabilizing $(A, B)$, the pair $\left(F, M_{1}\right)$ defined in (39) and (40), respectively, are $R$-reachable.

The major difficulty with existence type results of the form of Theorem 7 is that they are not really feasible as general purpose algorithms for control systems design. In the remainder of this section, we show that the design of control laws of the form (36) is highly tractable in an LMI setting (at a cost of sufficient but not necessary conditions for closed loop stability along the pass).

Introduce the matrices

$$
\hat{B}_{1}=\left[\begin{array}{c}
B \\
0
\end{array}\right], \quad \hat{B}_{2}=\left[\begin{array}{l}
0 \\
D
\end{array}\right] .
$$

Then, we have the following result which is simply Theorem 2 applied to the closed loop system.

Theorem 8: Suppose that a discrete linear repetitive process of the form described by (1) and (2) is subjected to a control law of the form (36). Then, the resulting closed-loop process is stable along the pass if $\exists$ symmetric matrices $P>0$ and $Q>0$ such that we get (44) shown at the bottom of the next page.

The difficulty with the matrix inequality of Theorem 8 is that it is nonlinear in its parameters. It can, however, be converted into the following result where each of the inequalities is a strict LMI with a linear constraint which also gives a formula for computing $K$ in (36).

Theorem 9: The condition of Theorem 8 is equivalent to the requirement that $\exists$ symmetric matrices $Y>0, Z>0$, and an arbitrary matrix $N$ such that the following LMI holds.

$$
\left[\begin{array}{ccc}
Z-Y & 0 & Y \hat{A}_{1}^{T}+N^{T} \hat{B}_{1}^{T} \\
0 & -Z & Y \hat{A}_{2}^{T}+N^{T} \hat{B}_{2}^{T} \\
\hat{A}_{1} Y+\hat{B}_{1} N & \hat{A}_{2} Y+\hat{B}_{2} N & -Y
\end{array}\right]<0 .
$$

$$
C_{c}\left(z_{1}, z_{2}\right):=\operatorname{det}\left[\begin{array}{cc}
I_{n}-z_{1}\left(A+B K_{1}\right) & -z_{1}\left(B_{0}+B K_{2}\right) \\
-z_{2}\left(C+D K_{1}\right) & I_{m}-z_{2}\left(D_{0}+D K_{2}\right)
\end{array}\right] \neq 0 \quad \forall\left(z_{1}, z_{2}\right) \in \bar{U}^{2}
$$


Also if (45) holds, a stabilizing $K$ for the control law (36) is given by

$$
K=N Y^{-1} \text {. }
$$

Proof: Apply the Schur's complement formula to (44), followed by the congruence transformation defined by $\operatorname{diag}\left(P^{-1}, P^{-1}, P^{-1}\right)$. Then introduce the substitutions $Z=P^{-1} Q P^{-1}>0, Y=P^{-1}>0$ to obtain

$$
\left[\begin{array}{ccc}
Z-Y & 0 & Y\left(\hat{A}_{1}^{T}+K^{T} \hat{B}_{1}^{T}\right) \\
0 & -Z & Y\left(\hat{A}_{2}^{T}+K^{T} \hat{B}_{2}^{T}\right) \\
\left(\hat{A}_{1}+\hat{B}_{1} K\right) Y & \left(\hat{A}_{2}+\hat{B}_{2} K\right) Y & -Y
\end{array}\right]<0 .
$$

Use of (46) now completes the proof.

\section{ROBUSTNESS-STATIC BOUNDARY CONDITIONS}

In this section, we develop an LMI approach to stability analysis in the presence of uncertainty in the process definition-a key problem area for which no substantial results currently exist. In particular, recall the definition of the augmented plant matrix $\hat{A}=\hat{A}_{1}+\hat{A}_{2}$ and define the so-called augmented plant input matrix as $\Psi=\left[\begin{array}{ll}B^{T} & D^{T}\end{array}\right]^{T}$. Then, we treat the case when these matrices are subject to additive perturbations defined as follows:

$$
\hat{A}_{p}=\hat{A}+\widehat{\Delta A}, \quad \Psi_{p}=\Psi+\Delta \Psi
$$

where

$$
\widehat{\Delta A}=\left[\begin{array}{cc}
\Delta A & \Delta B_{0} \\
\Delta C & \Delta D_{0}
\end{array}\right], \quad \Delta \Psi=\left[\begin{array}{c}
\Delta B \\
\Delta D
\end{array}\right] .
$$

Also, we assume that the uncertainties here have the following typical structure:

$$
[\widehat{\Delta A} \quad \Delta \Psi]=\left[\begin{array}{l}
H_{1} \\
H_{2}
\end{array}\right] F\left[\begin{array}{ll}
E_{1} & E_{2}
\end{array}\right]
$$

where the matrices on the right-hand side are of compatible dimensions.

Now, introduce the following matrices

$$
\begin{aligned}
& {\widehat{\Delta A_{1}}}^{=}\left[\begin{array}{cc}
\Delta A & \Delta B_{0} \\
0 & 0
\end{array}\right] \quad \widehat{\Delta A_{2}}=\left[\begin{array}{cc}
0 & 0 \\
\Delta C & \Delta D_{0}
\end{array}\right] \\
& \widehat{\Delta \Psi}_{1}=\left[\begin{array}{c}
\Delta B \\
0
\end{array}\right] \quad \widehat{\Delta \Psi}_{2}=\left[\begin{array}{c}
0 \\
\Delta D
\end{array}\right] .
\end{aligned}
$$

Then, we can write $\widehat{\Delta A}$ and $\Delta \Psi$ in the form

$$
\begin{aligned}
& \widehat{\Delta A}=\widehat{\Delta A}_{1}+\widehat{\Delta A}_{2}=\hat{H}_{1} F E_{1}+\hat{H}_{2} F E_{1} \\
& \Delta \Psi=\widehat{\Delta \Psi}_{1}+\widehat{\Delta \Psi}_{2}=\hat{H}_{1} F E_{2}+\hat{H}_{2} F E_{2}
\end{aligned}
$$

where $\hat{H}_{1}=\left[\begin{array}{ll}H_{1}^{T} & 0\end{array}\right]^{T}$, and $\hat{H}_{2}=\left[\begin{array}{ll}0 & H_{2}^{T}\end{array}\right]^{T}$. Note here that $H_{i}$ and $E_{i}, i=1,2$, are known matrices with constant entries and
$F$ is a possibly time varying matrix subject only to the constraint that $F^{T} F<I$.

The LMI sufficient condition for stability along the pass given in Theorem 2 applied in this case is equivalent to the existence of symmetric matrices $P>0$ and $Q>0$ such that

$$
\tilde{A}^{T} P \tilde{A}+\hat{Q}<0
$$

where

$$
\tilde{A}=\left[\begin{array}{ll}
\hat{A}_{1} & \hat{A}_{2}
\end{array}\right] \quad \hat{Q}=\left[\begin{array}{cc}
P-Q & 0 \\
0 & -Q
\end{array}\right]
$$

and we now have the following result which follows immediately from re-arranging (51).

Theorem 10: Discrete linear repetitive processes described by (1) and (2) whose defining matrices have the uncertainty structure defined above are stable along the pass if $\exists$ symmetric matrices $P>0, Q>0$ such that

$$
\left(\tilde{A}+\hat{H} \hat{F} \hat{E}_{1}\right)^{T} P\left(\tilde{A}+\hat{H} \hat{F} \hat{E}_{1}\right)+\hat{Q}<0
$$

where

$$
\hat{H}=\left[\begin{array}{ll}
\hat{H}_{1} & \hat{H}_{2}
\end{array}\right], \quad \hat{F}=I_{2} \otimes F, \quad \hat{E}_{1}=I_{2} \otimes E_{1}
$$

and $\otimes$ denotes the Kronecker product of two matrices.

Also it can be shown that, for any choice of $Q, \exists P>0$ such that (52) holds if, and only if, $\exists$ a scalar $\epsilon>0$ such that

$$
\left[\begin{array}{cc}
-P^{-1}+\epsilon \hat{H} \hat{H}^{T} & \tilde{A} \\
\tilde{A}^{T} & \epsilon^{-1} \hat{E}_{1}^{T} \hat{E}_{1}+\hat{Q}
\end{array}\right]<0 .
$$

Now, we are in a position to characterize stability along the pass under the uncertainty structure defined above. Here there are two cases of interest, the first of which is open loop and the second is closed loop under a control law of the form (36). Theorem 11 gives an LMI based sufficient condition for the former case and Theorem 12 for the latter.

Theorem 11: Discrete linear repetitive processes described by (1) and (2) whose defining matrices have the uncertainty structure defined above are stable along the pass if $\exists$ symmetric matrices $P>0$ and $Q>0$ and a real scalar $\varepsilon$ such that the LMI shown in (54) at the bottom of the next page holds.

Proof: Follows immediately on applying the Schur's complement formula to (52) and use of (53).

Theorem 12: Discrete linear repetitive processes described by (1) and (2) whose defining matrices have the uncertainty structure defined above are stable along the pass under the control law (36) with $K$ defined by (46) if $\exists$ a scalar $\epsilon>0$ and matrices $Y>0, Z>0$, and $N$ such that the LMI shown in (55) at the bottom of the next page holds.

Proof: This involves extensive, but routine manipulations and hence here we only give the main steps. The first step is to interpret (52) for the closed loop system obtained by applying

$$
\left[\begin{array}{cc}
\left(\hat{A}_{1}^{T}+K^{T} \hat{B}_{1}^{T}\right) P\left(\hat{A}_{1}+\hat{B}_{1} K\right)+Q-P & \left(\hat{A}_{1}^{T}+K^{T} \hat{B}_{1}^{T}\right) P\left(\hat{A}_{2}+\hat{B}_{2} K\right) \\
\left(\hat{A}_{2}^{T}+K^{T} \hat{B}_{2}^{T}\right) P\left(\hat{A}_{1}+\hat{B}_{1} K\right) & \left(\hat{A}_{2}^{T}+K^{T} \hat{B}_{2}^{T}\right) P\left(\hat{A}_{2}+\hat{B}_{2} K\right)-Q
\end{array}\right]<0
$$


(36) to the process. Then apply in turn Theorems $8-11$ to this system.

\section{CONTROLLER DESIGN—DYNAMIC BOUNDARY CONDITIONS}

We consider first the unit memory case and start with the application of a control law of the following form expressed in terms of the 1-D equivalent model (15)-(17) of a discrete unit memory linear repetitive process with dynamic boundary conditions

$$
\mathbf{U}(l)=-K \mathbf{Y}(l) .
$$

Note here that this control law (and the natural generalization to the nonunit memory case (see later in this section)) is defined only in terms of the output equation (that for updating $\mathbf{Y}(l)$ ) of the 1-D equivalent model. The reason for this is that the state equation in this 1-D equivalent model has no dynamic updating. Also, interpreting (18) for asymptotic stability in this case gives that this property holds if $\exists$ a symmetric matrix $W>0$ and $K$ such that

$$
\left[\begin{array}{cc}
-W & \Phi^{T} W-K^{T} \Delta^{T} W \\
W \Phi-W \Delta K & -W
\end{array}\right]<0 .
$$

This, however, is not in the LMI form since it involves the product of two matrices, $K$ and $W$, which have to be designed. We have, however, the following result which removes this problem.

Theorem 13: Suppose that a discrete linear repetitive process described by (1) and (3) is subject (via its 1-D equivalent model representation) to a feedback control law of the form (56). Then the resulting closed loop system is asymptotically stable if, and only if, $\exists$ a symmetric matrix $W>0$ and a matrix $N$ such that

$$
\left[\begin{array}{cc}
-W & W \Phi^{T}-N^{T} \Delta^{T} \\
\Phi W-\Delta N & -W
\end{array}\right]<0 .
$$

Also, if this condition holds, a stabilizing feedback controller is defined by $K=N W^{-1}$.
Proof: Follows immediately on applying a congruence transformation to (58) defined by $\operatorname{diag}\left(W^{-1}, W^{-1}\right)$ and then replacing $W^{-1}$ by $W$.

Write the control law (56) in the form

$$
u_{l}(p)=-\sum_{j=0}^{\alpha-1} K_{p j} v_{l}(j)
$$

or on, recovering the original process variables $y_{k}(p)$,

$$
u_{k+1}(p)=-\sum_{j=0}^{\alpha-1} K_{p j} y_{k}(j)
$$

where $K=\left[K_{p j}\right]_{j=0 \ldots \alpha-1, p=0 \ldots \alpha-1}$. Then, in physical terms, we see that control vector $u_{k+1}(p)$ applied at any point on a given pass computed using this law depends on the complete previous pass profile vector $y_{k}(p), 0 \leq p \leq \alpha-1$, and this could lead to implementation difficulties. To simplify the structure, we can require that in Theorem $13 W=\operatorname{diag}\left(W_{0}, W_{1}, \ldots, W_{\alpha-1}\right):=W_{p}, N=$ $\operatorname{diag}\left(N_{0}, N_{1}, \ldots, N_{\alpha-1}\right):=N_{p}$, where $W_{j}$ and $N_{j}$ are matrices of dimensions $m \times m$ and $r \times m$ respectively. Then the control law takes the form

$$
u_{k+1}(p)=-K_{p} y_{k}(p)=-N_{p} W_{p}^{-1} y_{k}(p)
$$

which introduces a considerable simplification in implementation terms since now the complete previous pass dependence has been replaced by a point to point dependence.

Another possible simplification is to require that the controller is constant along the pass, i.e., assuming that $W_{j}=$ $\tilde{W}, N_{j}=\tilde{N}$, which yields the controller

$$
u_{k+1}(p)=-\tilde{K} y_{k}(p)=-\tilde{N} \tilde{W}^{-1} y_{k}(p) .
$$

This requirement is, however, more strict and hence more difficult to satisfy than the previous one.

$$
\left[\begin{array}{ccccc}
-P & P \hat{A}_{1} & P \hat{A}_{2} & P \hat{H}_{1} & P \hat{H}_{2} \\
\hat{A}_{1}^{T} P & Q-P+\varepsilon E_{1}^{T} E_{1} & 0 & 0 & 0 \\
\hat{A}_{2}^{T} P & 0 & -Q+\varepsilon E_{1}^{T} E_{1} & 0 & 0 \\
\hat{H}_{1}^{T} P & 0 & 0 & -\varepsilon I & 0 \\
\hat{H}_{2}^{T} P & 0 & 0 & 0 & -\varepsilon I
\end{array}\right]<0
$$

$$
\left[\begin{array}{ccccccc}
-Y & \hat{A}_{1} Y+\hat{B}_{1} N & \hat{A}_{2} Y+\hat{B}_{2} N & \varepsilon \hat{H}_{1} & \varepsilon \hat{H}_{2} & 0 & 0 \\
Y \hat{A}_{1}^{T}+N^{T} \hat{B}_{1}^{T} & Z-Y & 0 & 0 & 0 & Y E_{1}^{T}+N^{T} E_{2}^{T} & 0 \\
Y \hat{A}_{2}^{T}+N^{T} \hat{B}_{2}^{T} & 0 & -Z & 0 & 0 & 0 & Y E_{1}^{T}+N^{T} E_{2}^{T} \\
\varepsilon \hat{H}_{1}^{T} & 0 & 0 & -\varepsilon I & 0 & 0 & 0 \\
\varepsilon \hat{H}_{2}^{T} & 0 & 0 & 0 & -\varepsilon I & 0 & 0 \\
0 & E_{1} Y+E_{2} N & 0 & 0 & 0 & -\varepsilon I & 0 \\
0 & 0 & E_{1} Y+E_{2} N & 0 & 0 & 0 & -\varepsilon I
\end{array}\right]<0
$$


It is also possible to re-formulate Theorem 13 into a more computationally attractive form by simplifying the form of underlying matrices. This first requires two intermediate results for the open loop case (Lemmas 6 and 7 below).

First write the matrix $\Phi$ of the 1-D equivalent model as $\Phi=$ $\tilde{\Phi}+\Theta J$, where the matrix $\tilde{\Phi}$ is obtained from (16) by setting $J_{i}=0, i=0,1, \ldots, \alpha-1$. Note that the matrix $\Theta J$ can be rewritten as $\Theta J=C A \mathcal{J}$ where

$\mathbb{C}=\left\|\operatorname{diag}\left\{I, A, A^{2}, \ldots, A^{\alpha-1}\right\}\right\| \operatorname{diag}\{C\}_{\alpha}$

$\mathrm{A}=\left\|\operatorname{diag}\left\{I, A, A^{2}, \ldots, A^{\alpha-1}\right\}\right\|^{-1} \operatorname{diag}\left\{I, A, A^{2}, \ldots, A^{\alpha-1}\right\}$ $\mathfrak{J}=\left[\begin{array}{ccc}J_{0} & \cdots & J_{\alpha-1} \\ \vdots & & \vdots \\ J_{0} & \cdots & J_{\alpha-1}\end{array}\right]$

where $\|\cdot\|$ denotes any convenient matrix norm. Hence, noting that $\|\mathbb{A}\|=1$ allows us to employ a result similar to Theorem 10.

Lemma 6: Discrete unit memory linear repetitive processes described by (1) and (3) are asymptotically stable if $\exists$ a symmetric matrix $W>0$ and a real scalar $\varepsilon>0$ such that

$$
\left[\begin{array}{cc}
-W^{-1}+\varepsilon \mathbb{C C}^{T} & \tilde{\Phi} \\
\tilde{\Phi} & \varepsilon^{-1} J^{T} J-W
\end{array}\right]<0
$$

Proof: This is a direct consequence of previous assumptions and Theorem 10.

Applying Schur complements and appropriate congruence transforms enables Lemma 6 to be restated in the following form which is more attractive in terms of onward analysis.

Lemma 7: Discrete unit memory linear repetitive processes described by (1) and (3) are asymptotically stable if $\exists$ a symmetric matrix $W>0$ and a real scalar $\varepsilon>0$ such that

$$
\left[\begin{array}{cccc}
-W & W \tilde{\Phi} & W \mathbb{C} & 0 \\
\tilde{\Phi}^{T} W & -W & 0 & \varepsilon J^{T} \\
\mathbb{C}^{T} W & 0 & -\varepsilon I & 0 \\
0 & \varepsilon J & 0 & -\varepsilon I
\end{array}\right]<0 .
$$

The following result uses the LMI of (65) to obtain the following alternative to Theorem 13.

Theorem 14: Consider a discrete linear repetitive process described by (1) and (3). Then this process is asymptotically stable under the action of the feedback law (56) if $\exists$ a symmetric matrix $W>0$, a matrix $N$ and a positive constant $\varepsilon$ such that

$$
\left[\begin{array}{cccc}
-W & \tilde{\Phi} W-\Delta N & \mathbb{C} \varepsilon & 0 \\
(\tilde{\Phi} W-\Delta N)^{T} & -W & 0 & W J^{T} \\
\mathbb{C}^{T} \varepsilon & 0 & -\varepsilon I & 0 \\
0 & J W & 0 & -\varepsilon I
\end{array}\right]<0 .
$$

Also if this condition holds, a stabilizing feedback controller is defined by $K=N W^{-1}$.

Proof: Follows in a similar manner to that of Theorem 13 for the closed-loop case, i.e., replace $\Phi$ by $\tilde{\Phi}-\Delta K$ and then apply the congruence transformation defined by $\operatorname{diag}\left(W^{-1}, W^{-1}, \varepsilon^{-1} I, \varepsilon^{-1} I\right)$. Finally, make the substitution $W^{-1} \rightarrow W, \varepsilon^{-1} \rightarrow \varepsilon$.
Finally, note that if the stronger property of stability along the pass is required, then the controller emerging from the asymptotic stability design must also satisfy (5) of Theorem 1 interpreted closed loop. As noted previously, this can be addressed using the LMI based analysis of Section III.

In order to apply the above analysis to the case of a discrete nonunit memory linear repetitive process with dynamic boundary conditions, the basic route is to first construct the 1-D equivalent model. Now, however, the dimension of the vector replacing $\mathbf{Y}(l)$ in (56) is considerably larger but all of the results given above for the unit memory case above generalize in a natural manner. Hence they are not explicitly given here except to note that $K$ in (56) now expands to $K=\left[\begin{array}{llll}K_{0} & K_{1} & \cdots & K_{\alpha-1}\end{array}\right]$.

\section{CONCLUSION}

This paper has developed an LMI based approach to stability along the pass of discrete linear repetitive processes-a distinct class of 2-D linear systems of both theoretical and applications interest. Much of the previous work on these processes had focused on stability theory and other systems theoretic properties such as controllability and observability and the general area of control systems design had been the subject of very little (in relative terms) work. In particular, there was a lack of an approach to stability analysis/tests which could also serve as a firm basis for control systems specification and design. Here, it has been shown that an LMI setting has very great potential in this general area.

This last claim has been justified by showing that LMI based stability analysis can be extended naturally from the simplest case of linear dynamics and static boundary conditions to include dynamic boundary conditions and also processes where the dynamics are singular along the pass in a well defined and relevant sense. Moreover, the stability analysis has been shown to be a systematic computationally attractive basis for the design of control laws, where here this has been demonstrated by the design of a particularly powerful control law, motivated from the ILC application area, combining current pass state feedback with feedforward action from the previous pass (or passes in the non unit memory case) for all the forms of discrete linear repetitive process dynamics considered. Also it has been shown that this LMI setting can be used to study the critical problem of stability analysis and controller design in the presence of uncertainty in the process model description (the beginnings of a robust control theory/design algorithms).

\section{REFERENCES}

[1] J. B. Edwards, "Stability problems in the control of multipass processes," Proc. Inst. Elect. Eng., vol. 121, no. 11, pp. 1425-1431, 1974.

[2] S. E. Benton, "Analysis and control of linear repetitive processes," Ph.D. dissertation, Dep. of Electron. Comp. Sci., Univ.of Southampton, Southampton, U.K., 2000.

[3] N. Amann, D. H. Owens, and E. Rogers, "Predictive optimal iterative learning control," Int. J. Control, vol. 69, no. 2, pp. 203-206, 1998.

[4] P. D. Roberts, "Stability analysis of iterative optimal control algorithms modeled as linear repetitive processes," in Proc. Inst. Elect. Eng., vol. 147, 2000, pp. 229-238.

[5] R. P. Roesser, "A discrete state-space model for linear image processing," IEEE Trans. Automat. Contr., vol. AC-20, pp. 1-10, Jan. 1975.

[6] E. Fornasini and G. Marchesini, "Doubly-indexed dynamical systems: State-space models and structural properties," Math. Syst. Theory, vol. 12, no. 6, pp. 59-72, 1978. 
[7] T. Kaczorek, "Generalized 2-D continuous-discrete linear systems with delay," Appl. Math. Comp. Sci., vol. 5, no. 3, pp. 439-454, 1995.

[8] E. Rogers and D. H. Owens, "Stability analysis for linear repetitive processes," in Lecture Notes in Control and Information Sciences Series, Berlin, Germany: Springer-Verlag, 1992, vol. 175.

[9] S. Boyd, L. E. Ghaoui, E. Feron, and V. Balakrishnan, "Linear matrix inequalities in systems and control theory," in SIAM Studies in Applied Mathematics. Philadelphia, PA: SIAM, 1994.

[10] E. Rogers, J. Lam, K. Galkowski, S. Xu, J. Wood, and D.H. Owens, "LMI based stability analysis for discrete linear repititive processes," in Proc. IEEE 40th Conf. Decision and Control, Orlando, FL, Dec. 2001.

[11] K. Galkowski, E. Rogers, and D. H. Owens et al., "1-D model based stability analysis for a class of 2D linear systems," in Mathematical Theory of Networks and Systems, A. Beghi et al., Eds. Padova, Italy: IL Poligrafo, 1998, pp. 165-172.

[12] J. L. Aravena, M. Shaffiee, and W. A. Porter, "State models and stability for 2-D filters," IEEE Trans. Circuits Syst., vol. CAS-37, pp. 1509-1519, Dec. 1982.

[13] E. Rogers, K. Galkowski, and D. H. Owens, "Control systems theory and applications for linear repetitive processes," in Lecture Notes in Control and Information Sciences Series. Berlin, Germany: Springer-Verlag, 2002 , to be published.

[14] K. L. Hsiung and L. Lee, "Lyapunov inequality and bounded real lemma for discrete-time descriptor systems," Proc. Inst. Elect. Eng., vol. 146, no. 4, pp. 327-332, 1999.

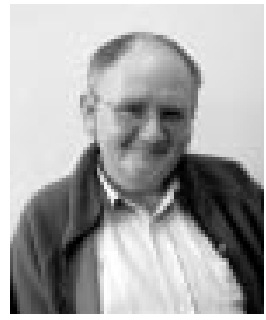

K. Galkowski received the M.S., Ph.D., and Habilitation degrees in electronics/automatic control from the Technical University of Wroclaw, Wroclaw, Poland in 1972, 1977, and 1994, respectively.

In October 1996, he joined the Technical University of Zielona Gora (now the University of Zielona Góra), Zielona Góra, Poland, where he holds the Professor position. Recently, he has been awarded a Visiting Professor position at the University of Southampton, Southampton, U.K. His research interests include multivariable $(\mathrm{nD})$ systems, repetitve processes-theory and applications, control and related numerical and symbolic algebra methods. $\mathrm{He}$ is an author/editor of three monographs/books and over 80 research texts published in the leading journals and proceedings of international conferences.

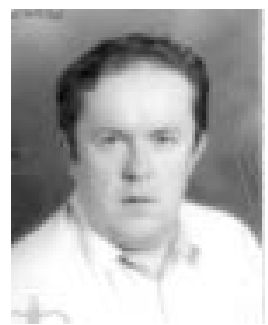

Eric Rogers was born in 1956 near Dungannon. Northern Ireland, U.K. He received the undergraduate degree in mechanical engineering from The Queen's University, Belfast, U.K., and the Ph.D. degree from The University of Sheffield, Sheffield, U.K., for a thesis in the area of multidimensional systems theory.

He was a Lecturer at The Queen's University from 1984 to 1987, and at the University of Strathclyde, Strathclyde, U.K., from 1988 to 1990 . He has been with The University of Southampton, U.K., since 1990, where he is currently Professor of Control Systems Theory and Design in The Department of Electronics and Computer Science. His current major research interests include multidimensional systems theory and applications, with particular emphasis on behavioral systems theory approaches and systems with repetitive dynamics, iterative learning control, flow control, and active control of microvibrations. He is currently the Editor of The International Journal of Control, an Associate Editor of Multidimensional Systems and Signal Processing, and Joint Editor of The Taylor and Francis Research Book Series on Systems and Control. In addition, he has served extensively on IEEE, IFAC, and IEE technical committees and acted as a consultant to numerous companies and government agencies in the U.K. and abroad.

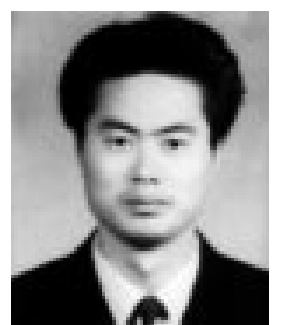

S. Xu was born in 1968 in Zhejiang Province, China. He received the B.Sc. degree from Hangzhou Normal University, Hangzhou, China, the M.Sc. degree from Qufu Normal University, Qufu, China, and the Ph.D. degree from Nanjing University of Science and Technology, Nanjing, China, in 1990, 1996, and 1999, respectively

From 1999 to 2000, he was a Research Associate in the Department of Mechanical Engineering, University of Hong Kong, Hong Kong. From December 2000 to November 2001, he was a Postdoctoral Researcher in CESAME at the Universitè catholique de Louvain, Louvain-la-Neuve, Belgium. He is currently a Postdoctoral Researcher in the Department of Electrical and Computer Engineering, University of Alberta, Edmonton, Canada. His research interests include robust filtering and control, singular systems, time-delay systems, multidimensional systems and nonlinear systems.

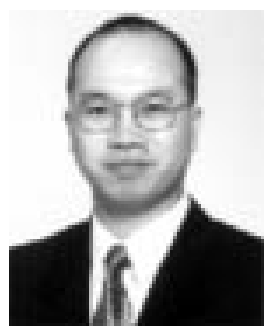

J. Lam (S'86-M'86-SM'99) received the first class B.Sc. degree in mechanical engineering from the University of Manchester, Manchester, U.K. in 1983, and the M.Phil. and Ph.D. degrees in the area of control engineering, from the University of Cambridge, Cambridge, U.K., in 1985 and 1988, respectively.

His postdoctoral research was carried out in the Australian National University between 1990 and 1992. He has held faculty positions at the now City University of Hong Kong, Hong Kong, and the University of Melbourne, Melbourne, Australia. He is currently an Associate Professor in the Department of Mechanical Engineering, the University of Hong Kong, and is holding a Concurrent Professorship at the Northeastern University, Guest Professorhsip at the Huazhong University of Science and Technology, and Consultant Professorship at the South China University of Technology. His research interests include model reduction, delay systems, generalized systems, multidimensional systems, robust control, fault detection, and reliable control.

Dr. Lam is a Chartered Mathematician, a Fellow of the Institute of Mathematics and Its Applications (U.K.). He is an Associate Editor of the Asian Journal of Control. He was awarded the Ashbury Scholarship, the A. H. Gibson Prize and the H. Wright Baker Prize for his academic performance during his undergraduate study and is a Scholar (1984) and Fellow (1990) of the Croucher Foundation.

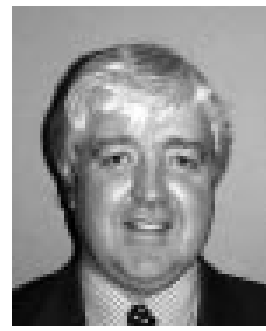

D. H. Owens is Head of the Department of Automatic Control and Systems Engineering at the University of Sheffield, Sheffield, U.K. He has previously held senior appointments as Professor of Engineering Mathematics and Mechanical Engineering at the University of Strathclyde in Scotland from 1985 to 1990 , and as Professor and Head of the School of Engineering and Computer Science at the University of Exeter, Exeter, U.K., from 1990 to 1999. His research interests include theoretical aspects of systems modeling, controller design and control algorithm development and he has experience of applications in areas such as the nuclear industry, coal-fired power plant, building systems control and pneumatic conveying systems. This work has lead to many major research grants, three textbooks and over 380 technical publications. He is currently a member of the IEE Council and the IMechE Machine Systems, Computing and Control Committee. $\mathrm{He}$ is the Chairman of the United Kingdom Automatic Control Council for the period 1999-2002 and is also an Independent Member of the U.K. Health and Safety Executive Nuclear Safety Committee. 